\title{
[Full title]
}

Meta-Analyses in Survey Methodology: A Systematic Review

[Author list, * denotes the corresponding author]

Gregor Čehovin* Institution: Faculty of Social Sciences, University of Ljubljana. Street address: Kardeljeva Ploščad 5, 1000 Ljubljana, Slovenia. Phone number: +386 15805 313. Email address: gregor.cehovin@fdv.uni-lj.si.

Michael Bosnjak Institution: ZPID - Leibniz Institute for Psychology Information. Street address: Universitätsring 15, 54296 Trier, Germany. Phone number: +49 0651 2012706. Email address: director@leibniz-psychology.org.

Katja Lozar Manfreda Institution: Faculty of Social Sciences, University of Ljubljana. Street address: Kardeljeva Ploščad 5, 1000 Ljubljana, Slovenia. Phone number: +386 15805114. Email address: katja.lozar@fdv.uni-lj.si.

\section{[Running Header]}

Meta-Analyses in Survey Methodology

\section{[Word count]}

Abstract: 235 .

Text: 5,346 (including footnotes; excluding figures, tables, references, and appendices).

This article was originally published in Public Opinion Quarterly, 82(4): 641-660 by Oxford University Press. The publisher's version of this article is available at: https://doi.org/10.1093/poq/nfy042.

Suggested citation:

Gregor Čehovin, Michael Bosnjak, Katja Lozar Manfreda, Meta-Analyses in Survey Methodology: A Systematic Review, Public Opinion Quarterly, Volume 82, Issue 4, Winter 2018, Pages 641-660, https://doi.org/10.1093/poq/nfy042. 


\section{[a. Author affiliation information]}

GREGOR ČEHOVIN is a PhD student and Young Researcher at the Faculty of Social Sciences, University of Ljubljana, Slovenia.

MICHAEL BOSNJAK is director of ZPID - Leibniz Institute for Psychology Information, Trier, Germany, and full professor of Psychology at the University of Trier, Germany.

KATJA LOZAR MANFREDA is an associate professor of Statistics at the Faculty of Social Sciences, University of Ljubljana, Slovenia.

\section{[b. Acknowledgements]}

The authors acknowledge the contributions of Professor Sandra Torres (Uppsala University) for her insightful comments, and PhD student Chiara Respi (University of Milano-Bicocca) for independently coding a random subset of manuscripts included in the systematic review. The research results were achieved as part of Gregor Čehovin's Young Researcher fellowship, financed from the national budget by a contract between the Slovenian Research Agency and the Faculty of Social Sciences, University of Ljubljana.

\section{[e. Corresponding author contact information]}

*Address correspondence to Gregor Čehovin, Faculty of Social Sciences, University of Ljubljana, Kardeljeva ploščad 5, 1000 Ljubljana, Slovenia; email: gregor.cehovin@fdv.uni-lj.si. 


\section{[Abstract]}

Meta-analytic techniques have become the standard methods for aggregating the results from thematically related studies in the behavioral, health, and economic sciences. To analyze the state of the art of using meta-analyses in survey methodology, previous meta-analyses are systematically identified and classified according to the thematic areas the analyses address. This is followed by identifying gaps in research (i.e., areas where there are few or no existing metaanalyses) and investigating potential avenues for future meta-analyses in this field. The findings are based on a systematic search of two bibliographic harvesters (together covering 265 bibliographic databases), which yielded 54 eligible manuscripts reporting 60 meta-analytic studies and 91 effect sizes. To identify the thematic areas, the effect sizes are structured according to seven categories of the total survey error (TSE). Characteristics of primary studies that potentially influence the variability of meta-analytic findings are also considered, such as the survey mode, questionnaire design, and sample characteristics. The results show that the thematic areas of these meta-analyses cover only two of the seven TSE categories: measurement and nonresponse error. Thematic areas in the remaining TSE categories are not covered, and the key practical implication is that there are gaps in current research. Regarding the methodology of existing meta-analyses in the survey methodology field, there are fundamental differences in reporting styles in terms of transparency and replicability, calling for a concerted effort to develop meta-analytic reporting standards for survey methodology. 


\section{Introduction}

Under the evidence-based paradigm, meta-analyses play an important role and are often used to inform policy or practice. Formally, a meta-analysis is a statistical analysis of a large collection of results from primary studies to integrate their findings (Glass 1976).1 It produces an overall summary of empirical knowledge for a specific topic and allows for the generalization of findings (Littell, Corcoran, and Pillai 2008; Koricheva and Gurevitch 2013). As a meta-analysis can quantify the size, direction, and/or strength of an effect, as well as cancel out sampling errors of individual studies, a higher level of precision and validity is typically attributed to meta-analytic findings (Bosnjak 2017). Correspondingly, evaluations of the epistemological strength of evidence place meta-analyses and systematic reviews of randomized controlled experiments at the highest level of evidence, situating these approaches above randomized controlled experiments, non-randomized controlled experiments, and quasi-experimental studies (Houser and Oman 2010; Dearholt and Dang 2012).

The health sciences were the first to replace case studies and theoretical reasoning with findings based on the systematic syntheses of high-quality experimental research (Bosnjak 2017), with numerous meta-analyses conducted for the same topic by different researchers (Haidich 2010). Borman and Grigg (2009) also showed a high number of meta-analytic papers in the field of psychological research. In survey methodology, in contrast, there are fewer meta-analyses, which

1 A summary of the meta-analytic concepts employed in this paper is provided in Part A of the Online Appendix, to introduce the concepts to readers who are unfamiliar with meta-analysis. 
could be partially explained by the context, as it is a field with fewer scholars and publications compared to the health and psychological sciences. This adds to the importance of stimulating additional meta-analyses in survey methodology as one of the scientific disciplines that aim to generate and use the best empirical evidence to guide actions.

The primary objective of this paper is threefold. First, this study aims to systematically identify previous meta-analyses in survey methodology. Second, it aims to classify the thematic areas addressed by the identified meta-analyses according to the seven categories of the total survey error (TSE) framework, among other variables that may explain the variability of meta-analytic findings in survey methodology. Third, the systematic review aims to highlight the gaps in current research and may therefore point to avenues for future meta-analyses.

\section{Research Questions}

\section{RQ1: WHICH RESEARCH QUESTIONS WERE ADDRESSED IN META-ANALYSES?}

To classify the identified meta-analyses, the study uses the structure of the total survey error (TSE) paradigm, which covers all types of systematic error (bias) and random error (variance) that occur in surveys2. TSE is central in survey methodology, because researchers must be diligent in identifying the major sources of survey error and must make design and estimation choices that minimize survey error (Groves et al. 2009; Dillman, Smyth, and Christian 2014).

2 See Biemer (2010) and Groves and Lyberg (2010) for a summary of the error sources incorporated in the total survey error paradigm, as well as Groves et al. (2009) for a discussion on research practices pertaining to different components of total survey error. 
Accordingly, the thematic areas of meta-analyses are structured into the seven TSE dimensions: specification, measurement, processing, coverage, sampling, nonresponse, and adjustment error (Groves et al. 2009; Biemer 2010). In addition, the meta-analyses are structured according to the type of intervention and the moderator variables of meta-analyses, which correspond to the characteristics of primary studies that may influence the variability of meta-analytic findings (e.g., data quality, contacting protocols, and questionnaire design).

\section{RQ2: WHAT GAPS BETWEEN THE RESEARCH QUESTIONS COULD HAVE BEEN ADDRESSED AND WERE ACTUALLY ADDRESSED?}

The two sets of dimensions of the classified meta-analyses (the TSE framework crossed with intervention variables and the TSE framework crossed with non-experimental moderators) are analyzed to identify specific research gaps, areas in which there are few or no meta-analyses available. The findings are critically discussed to demonstrate opportunities for future metaanalyses in survey methodology and outline their limitations. 


\section{Method for the Systematic Review and Description of the Included}

\section{Manuscripts}

\section{LITERATURE SEARCH STRATEGY AND SOURCES}

A search using two bibliographic harvesters, DiKUL3 and CLICsearch4, was performed between January 2017 and January 2018 to identify meta-analytic studies in the survey methodology field published in the scientific literature. The harvesters include 265 bibliographic databases, such as EBSCO, Google Scholar, Jstor, Medline, ProQuest, SAGE, ScienceDirect, Sociological Abstracts, and Web of Science. The searches were aimed at publications related to the keywords "meta-analysis" and "survey" within the title, abstract, and full text, according to singular, plural, and possessive combinations (see Part B of the Online Appendix for full lists of databases and exact search queries). Keywords that appeared in partial forms and in any combination with other words or phrases were also taken into account by the harvesters (e.g., web surveys, metaanalysis-based, and meta-analytic). The search was limited to manuscripts with the full text in the English language without limits for the publication date, and the first potentially eligible result was from 1983. Because the final search was conducted in January 2018, this determined our cutoff date as covering publications until the end of 2017. Unpublished studies and grey literature were not additionally explored or included.

3 DiKUL, Digital Library of University of Ljubljana, http://dikul.uni-lj.si.

${ }_{4}$ CLICsearch, Bush Memorial Library, Hamline University, https://clicsearch.hamline.edu/primoexplore/search?vid=HAMLINE. 


\section{INCLUSION AND EXCLUSION CRITERIA}

In addition to the basic criteria regarding the date (no limit) and language (English) of the publication, duplicates were excluded. Besides duplicates in the form of repeated bibliographic results referring to the same manuscripts, also excluded were studies that reported identical results and were published with basically the same content in different publications (e.g., a journal article published again as a book chapter). Furthermore, specific inclusion criteria were used, and the first inclusion criterion specified that the study was a meta-analysis. This excluded studies that (1) did not use meta-analytic methods to analyze results but instead used basic analyses that did not calculate an effect size; (2) analyzed a collection of primary studies subjectively (e.g., a narrative review of literature or vote counting methods that only compared the number of positive studies with the number of negative ones); (3) were not based on a systematic review of studies in the literature that investigate a specific research problem, but instead used a non-systematic approach to primary study selection, or selected a subjective and non-representative sample of specific studies (e.g., limiting the scope to only five studies performed by the same author); and (4) presented a discursive approach without actually conducting a meta-analysis, e.g., only a general methodological discussion on meta-analyses.

The second inclusion criterion required that the meta-analysis was a study in survey methodology. This demanded that the primary studies in the meta-analysis were quantitative research studies employing survey questionnaires. For this aspect, data collection modes were classified as direct (e.g., face-to-face) or indirect (e.g., telephone, mail, web surveys), depending on the contact with the respondent as suggested by Biemer and Lyberg (2003). Studies that used any combinations of these modes (mixed-mode surveys) were also included as classified as such. Meta-analyses in 
which the primary studies did not involve contact with the respondent were excluded (i.e., direct observation, computer-assisted data entry, administrative records, and electronic data interchange). Furthermore, the research problem of the primary studies included in the metaanalysis had to be in some way related to survey methodology research; that is, related to the study of the design, collection, processing, and analysis of surveys in relation to the cost and quality of the survey estimates as described by Groves et al. (2009).

The last point above meant including all the meta-analytic studies with research questions related to survey methodology as a discipline focusing on the quality of surveys, studying representation or measurement aspects of surveys, regardless of the studies' substantive field of origin. In addition to including the social and behavioral sciences, it also includes the health, organizational, marketing, and business sciences. Among the exclusions were meta-analyses that contained primary studies employing survey questionnaires, but with research questions related not to survey methodology, but instead to certain substantive questions-e.g., the likelihood of seeking health information online according to gender (Hallyburton and Evarts 2014) or associations of cigarette smoking and menthol cigarette use with all-cause, cancer, and cardiovascular risk (Jones, Tellez-Plaza, and Navas-Acien 2013). Studies employing survey questionnaires thus had to investigate at least some aspect of survey methodology to be included, for example the difference in health state valuations according to the survey mode (Peeters and Stiggelbout 2010). Following the above-described inclusion criteria meant that some metaanalyses were missed, as only published manuscripts were investigated. On the other hand, this approach ensured that the systematic review is based on manuscripts that provide sufficient conceptual and methodological quality of the meta-analysis in the full text. 


\section{STUDY SELECTION}

In total, 54 manuscripts reporting on at least one performed meta-analytic study with a theme related to survey methodology (from now on referred to as meta-analysis) met the inclusion criteria. The search process initially yielded 1,090 results, and the selection process included deduplication based on bibliographic data, the appraisal of abstracts, and the appraisal of the fulltext papers. The identification of duplicates revealed 261 duplicate results among the initial 1,090 results. The PRISMA flowchart (Moher et al. 2009) in Figure 1 illustrates the selection process that resulted in 54 eligible manuscripts.

\section{[FIGURE 1 ABOUT HERE]}

After deduplication, the abstracts of the remaining 799 manuscripts were evaluated in accordance with the inclusion criteria. If it was possible to identify a manuscript as ineligible beyond any doubt, it was excluded. In all other cases, manuscripts were scheduled for full-text evaluation. During the abstract appraisal, 660 results were excluded, and 139 manuscripts remained. The full-text content of the remaining 139 manuscripts was evaluated according to the inclusion criteria. Of the 139 manuscripts, 85 were excluded; 54 were eligible, consisting of three book sections (i.e., de Leeuw and van der Zouwen 1988; de Leeuw 1992; Mavletova and Couper 2015) and 51 journal papers (the full lists are in Parts C and D of the Online Appendix).

\section{DATA EXTRACTION AND SYNTHESIS}

After examining the full text of the 54 manuscripts, four manuscripts were found to contain more than one meta-analytic study (i.e., Lensvelt-Mulders et al. 2005; Gnambs and Kaspar 2016; Shih and Fan 2007; Tourangeau and Yan 2007). These were coded as separate meta-analytic studies 
because they were performed on thematically different sets of primary studies. This means that data for 60 meta-analytic studies from the 54 eligible manuscripts were extracted. In addition, effect sizes were coded as separate if a meta-analytic study reported more than one effect size, and the 60 meta-analytic studies reported 91 summary effect sizes (see Online Appendix Table A3).

All three authors of this paper collaborated to define the data structure of the coding and extraction process and resolved questions through discussion. The terminology in ten of the 54 manuscripts was sometimes not completely unambiguous for the purpose of classification and additional discussions were held among the authors until questions were unanimously resolved. The first author coded and extracted data that can be assessed objectively. They include study bibliographic descriptors, data related to reported meta-analytic studies (i.e., effect sizes, intervention variables, and moderators), and the results of the literature search and selection process in the meta-analyses.

The studies were then categorized as experimental or non-experimental meta-analyses.5 Their effect sizes were structured according to the seven dimensions of the TSE paradigm (e.g., Biemer 2010; Groves and Lyberg 2010), while the intervention and non-experimental moderator variables were structured according to survey-related characteristics that are under vs. not under control of the researcher (e.g., Groves and Couper 1998; Vehovar et al. 2002). Because this

5 Experimental and non-experimental meta-analyses were classified separately because of the fundamentally different approach at the outset: one begins with an investigation of an intervention variable, and the other starts with a set of non-experimental moderators (see Part A of the Online Appendix). 
process is partially subjective, the coding and extraction of the data were independently performed by another colleague for a random subset of 11 manuscripts. Differences were addressed via discussion, which took place in 11 out of 46 coding decisions, and agreement was reached in all instances. The intercoder reliability for the random subset of 11 manuscripts was initially 76 percent and reached 100 percent after discussion, expressed as the proportion of coding decisions that reached agreement among all coding decisions (e.g., Cho 2008).

\section{DESCRIPTION OF MANUSCRIPTS INCLUDED IN THE SYSTEMATIC REVIEW}

Regarding the reporting employed in meta-analyses, various standards have been established, such as the Preferred Reporting Items for Systematic Reviews and Meta-Analyses (PRISMA) Statement6 in 2009 and the Meta-Analysis Reporting Standards (MARS)7 Appendix of the Publication Manual by the American Psychological Association (2009). Differences exist in how closely such standards are followed in meta-analyses, and how several key stages of the metaanalytic process are reported (the literature search, selection strategy, and data extraction) is described in this paper. Table 1 presents the number of meta-analysis manuscripts across three levels of reporting8 and shows the fundamental differences in the transparency and replicability of the meta-analyses in survey methodology.

\section{[TABLE 1 ABOUT HERE]}

6 PRISMA Statement consisting of the checklist and flow diagram, http://prisma-statement.org/.

7 MARS, http://www.apastyle.org/manual/related/JARS-MARS.pdf.

8 The frequencies of the reporting level are summarized in Table 1, while the reporting level for each meta-analysis is provided in Online Appendix Table A3. 
Overall, only 16.7 percent of the meta-analysis manuscripts employed a comprehensive approach to documenting, which was informative enough to produce and include a complete PRISMA or comparable chart. A minimal approach to documenting was used in 40.7 percent of the manuscripts, which reported information on only some stages of the selection process (e.g., only the number of initial results and the number of eligible primary studies, but nothing about the

process in between). This minimal approach to documenting did not provide enough information for a PRISMA or comparable diagram. In 42.6 percent of the manuscripts, an insufficient approach to documenting was used, as only the number of eligible primary studies was reported. Among the insufficient and minimal reporting, of course, many meta-analyses were conducted in the years before reporting standards, such as PRISMA or MARS, were elaborated in detail (e.g., Armstrong and Lusk 1987; Yammarino, Skinner, and Childers 1991; Hopkins and Gullickson 1992; Church 1993, among others). Manuscripts that complied the most with the PRISMA statement were published only in 2014 or later, and only a few of such manuscripts were published before 2010 (Online Appendix Table A3).

\section{[TABLE 2 ABOUT HERE]}

Table 2 summarizes the type of the design, as well as the approaches to effect size models and measures of the 91 meta-analytic summary effect sizes found in the 60 meta-analytic studies. More than three fifths (61.5 percent) of the effect sizes were analyzed using the random-effects model (REM), and in the remaining effect sizes (38.5 percent), the fixed-effect model (FEM) was implemented. Differences between the models are discussed in the first section, but it is beyond the scope of this paper to evaluate whether the model selection was appropriate. In addition, the 
REM was the prevalent model for the effect sizes in experimental as well as non-experimental meta-analyses. The effect sizes that used the REM were from meta-analyses that were newer on average (mean age of 9.4 years) than those in meta-analyses that used the FEM (mean age of 21.2 years).

\section{[TABLE 3 ABOUT HERE]}

A comparison of the measures of effect size (Table 3) used in meta-analyses shows that odds/risk ratios were the most common (42.9 percent), followed by the mean difference 9 (31.9 percent), standardized regression coefficient (14.3 percent), and Pearson correlation coefficient (11.0 percent). Among the different effect size measures, odds/risk ratios were on average found in newer meta-analyses. In addition, odds/risk ratios were the most common effect size measure in experimental as well as non-experimental meta-analyses.

\section{Findings}

\section{RESEARCH QUESTIONS ADDRESSED IN PREVIOUS META-ANALYSES}

The results show that the thematic areas of the effect sizes of the meta-analyses cover only two TSE dimensions: measurement error and nonresponse error. This is illustrated in Online Appendix Tables A5 and A6, which classify the experimental and non-experimental metaanalyses from the systematic review according to the thematic areas of the 54 manuscripts'

9 This included standardized and non-standardized mean difference. 
research questions. A simpler illustration of Online Appendix Tables A5 and A6 is provided in Tables 4 and 5 below10. An individual cell in Tables 4 and 5 lists the frequency of the effect size that corresponds to one particular combination of one TSE dimension of the manuscript's research questions crossed with intervention/non-experimental moderator variables. The latter are structured according to several survey design characteristics that are under vs. not under the control of the researcher (e.g., Groves and Couper 1998; Vehovar et al. 2002). In addition, cells of combinations that do not make sense within the framework of survey methodology are marked with a light grey diagonal pattern.

\section{[TABLE 4 ABOUT HERE]}

\section{[TABLE 5 ABOUT HERE]}

Based on Online Appendix Tables A5 and A6, the intervention and non-experimental moderator variables investigating survey design characteristics under the researcher's control are discussed next. The manuscripts that cover multiple subcategories of survey design characteristics (e.g., incentives and sponsorship under contacting protocols) or multiple TSE dimensions are only counted once when reporting the overall number of manuscripts addressing a main category. The findings show that contacting protocols are among the variables that receive the most attention in past meta-analyses, as they are investigated by 26 manuscripts (37 effect sizes), comprising

10 The effect sizes that cover multiple subcategories of survey design characteristics are counted only once in Tables 4 and 5. Online Appendix Tables A5 and A6 report the exact manuscript, study, and effect size IDs corresponding to a particular cell instead of only the frequencies that appear in Tables 4 and 5 . In addition, the structure of intervention/non-experimental variables has one more level than what is shown in Tables 4 and 5. 
experimental (seven manuscripts, 16 effect sizes) and non-experimental (19 manuscripts, 21 effect sizes) meta-analyses. However, the manuscripts almost exclusively (except for two manuscripts) deal with the question of how some dimensions of contacting protocols affect the nonresponse issues (as opposed to measurement issues).

Both in experimental and non-experimental meta-analyses, the most attention within contacting protocols related to nonresponse is given to incentives (17 manuscripts, 23 effect sizes), followed by delivery and contacting method (13 manuscripts, 18 effect sizes), follow-up contact and reminders (12 manuscripts, 12 effect sizes), pre-notification (nine manuscripts, 10 effect sizes), sponsorship (nine manuscripts, 10 effect sizes), the personalization of survey invitations (seven manuscripts, eight effect sizes), and characteristics related to anonymity, confidentiality, and data security (seven manuscripts, seven effect sizes). The only two manuscripts on the effect of contacting protocols on measurement both analyze the effect of delivery and contacting methods (three effect sizes).

The mode of administration is the second main category that is covered by both a number of experimental (12 manuscripts, 21 effect sizes) as well as non-experimental meta-analyses (14 manuscripts, 22 effect sizes). These meta-analyses sum up to 26 manuscripts and 43 effect sizes. The third main category of survey design characteristics that receives the most attention in past meta-analyses comprises questionnaire design-related characteristics. The latter are investigated by 27 manuscripts and 32 effect sizes in total and comprise mostly non-experimental metaanalyses (22 manuscripts, 26 effect sizes) and only a few experimental studies (five manuscripts, six effect sizes). More specifically, these five experimental meta-analysis manuscripts focus on the effect of some aspects of the general format of the questionnaire (structure, length, and visual 
formatting) and the format of the questions. On the other hand, the non-experimental metaanalyses under questionnaire design investigate the general format of the questionnaire (12 manuscripts, 14 effect sizes), topic content (12 manuscripts, 14 effect sizes), topic salience (seven manuscripts, eight effect sizes), and topic sensitivity (seven manuscripts, eight effect sizes).

The above-discussed contacting protocols, mode of administration, and questionnaire design characteristics receive the most attention, as each is covered by more than 25 meta-analytic manuscripts. Two more main categories of survey design characteristics are investigated by experimental as well as non-experimental meta-analyses, but to a slightly lesser extent. First, the target population type is analyzed by 17 manuscripts and 22 effect sizes, comprising mostly nonexperimental meta-analyses (15 manuscripts, 20 effect sizes) and two experimental meta-analytic manuscripts (two effect sizes). Second, the survey setting (e.g., individual vs. group administration, standardized vs. non-standardized environment) receives attention from 11 manuscripts and 16 effect sizes, again comprising mainly non-experimental meta-analyses (nine manuscripts, 12 effect sizes) and two experimental ones (two manuscripts, four effect sizes).

The remaining main categories of survey design characteristics are addressed by fewer metaanalyses, which are exclusively non-experimental. These are data quality (e.g., survey burden, item nonresponse, and response quality; six manuscripts, seven effect sizes), and sample characteristics (six manuscripts, seven effect sizes). Non-experimental meta-analyses also investigate some factors that are not directly related to survey design, which are structured under the category other (eight manuscripts, nine effect sizes). These factors include a study's basic descriptors, such the date of the survey. Lastly, under survey-related characteristics that are not 
under the researcher's control, only several non-experimental meta-analyses cover national specifics in relation to the region of the study (five manuscripts, six effect sizes).

\section{RESEARCH AVENUES FOR FUTURE META-ANALYSES}

Based on the above discussion of research questions covered by past meta-analyses, blind spots are discussed next to point to issues that could have been addressed with a meta-analysis but have not received attention. These issues are shown as (almost) empty cells in Online Appendix Tables A5 and A6, meaning that few or no meta-analyses are currently available for those combinations of TSE framework dimensions crossed with intervention and non-experimental moderator variables. The first stream of research gaps is linked to the five TSE dimensions that have not been addressed as research questions by meta-analyses: specification, processing, coverage, sampling, and adjustment error. Therefore, survey design characteristics in combination with any of these dimensions represent opportunities for future meta-analytic research. The second stream of research gaps exists within the thematic areas of nonresponse and measurement error that were addressed as research questions by meta-analyses in the past but not in their entirety.

However, before several examples of these gaps are discussed, some reservations are presented. As mentioned, certain combinations of intervention/non-experimental moderator variables and effect sizes do not make sense within the framework of survey methodology, and such cells in Online Appendix Tables A5 and A6 are marked with a light grey diagonal pattern. In addition, the full scope of the blind spots is theoretical and aimed at further popularizing meta-analyses in survey methodology, while the actual implementation is limited by a sufficient number of primary studies. This number has to be assessed beforehand by researchers, and performing a 
power analysis 11 can provide additional insight into how many studies are expected to be required for a particular meta-analysis. For several of the theoretical opportunities for future metaanalyses, there might not be enough primary studies at this point, and the power of meta-analysis may be too low, especially to detect small effects. It is beyond the scope of this paper to systematically describe all possible avenues for future meta-analyses, based on the combinations of survey design characteristics and topics of effect sizes, as listed in Online Appendix Tables A5 and A6. Instead, some obvious examples are provided, as they are derived from theoretical reasoning. However, information on the number or a list of possible primary studies that could be used for such a meta-analysis is not given here.

Let us begin with the first stream of blind spots, that is, specification, processing, coverage, sampling, and adjustment error TSE dimensions, which have not been addressed with research questions of meta-analyses. Among the combinations that would make sense, one potential example is the effect of weighting (a design characteristic under the researcher's control) on adjustment error, such as the primary study by Vehovar, Lozar Manfreda, and Batagelj (1999). An example referring to survey design characteristics not under the researcher's control is how the country or region (country specifics) of the target population affects the coverage error.

11 Power analysis analyzes the a priori power of the summary effect size test and aims to answer whether there is a sufficiently high likelihood that the meta-analysis will yield a statistically significant result. Under the FEM, power analysis refers to the cumulative sample size across primary studies, among other factors. Under the REM, it also depends on the number of primary studies, as the between-study variance is an additional error source (Borenstein et al. 2009; Pigott 2012). 
Again, an example of an eligible primary study is Mohorko, De Leeuw, and Hox's (2013) study. A meta-analysis of similar studies for different survey questions would be beneficial.

The second stream of gaps is related to the TSE dimensions of measurement and nonresponse error that have been addressed with research questions of meta-analyses, but they did not take into account all possible survey design characteristics of our classification. First, several gaps can be exposed that are present under both dimensions. The most striking gap includes all the survey design characteristics that are not under the researcher's control (with the exception of country specifics that are included in non-experimental meta-analyses as a non-experimental moderator variable), although some primary studies might exist. An example is a meta-analysis of studies analyzing the effect of urbanicity on unit nonresponse rates, and an example of an eligible primary study is Goyder, Lock, and McNair's (1992) paper. Another similar blind spot exists for the type of survey, which comprises variables referring to panel, cross-sectional, and longitudinal surveys; the primary study example is Brüggen, van den Brakel, and Krosnick's (2016) paper. Interviewer attributes and behavior (related to socio-demographic characteristics, experience, and expectations) represent another gap under measurement and nonresponse error.

Within the nonresponse error dimension specifically, none of the previous meta-analyses addressed the format of questions (it was investigated by only two manuscripts in combination with the measurement dimension). Finally, within the measurement error dimension specifically, several gaps are present. Under questionnaire design, topic salience represents a gap for the measurement error dimension. Under contacting protocols, these research gaps include prenotification, follow-up and reminders, sponsorship and researcher, the personalization of invitations, incentives, as well as anonymity, confidentiality, and data security. This shows that 
almost all the meta-analyses that address contacting protocols do so in combination with the nonresponse error dimension, and an opportunity exists for future meta-analyses to examine this issue in relation to the measurement error dimension.

\section{Discussion and Conclusions}

This paper examined the state of the art of meta-analyses in survey methodology based on the identified 54 eligible meta-analytic manuscripts. Comparing the number of identified metaanalyses to a scientific field, such as psychology, suggests that there are fewer meta-analyses available in survey methodology. For example, Borman and Grigg's (2009) review identified that the Psychological Bulletin journal alone published 74 meta-analytic papers between 1985 and 1994 and 60 between 2000 and 2005. In contrast, survey methodology is an emerging scientific field, with fewer scholars and publications available. In addition, the survey methodology has only recently developed as a unified field and is inherently multidisciplinary (Groves et al. 2009). Consequently, it is not surprising that this systematic review identified meta-analyses investigating issues related to survey methodology that were published not just in survey methodology journals, but also journals primarily focused on other fields, such as the British Medical Journal, Evaluation \& the Health Professions, Educational and Psychological Measurement, Psychological Bulletin, Journal of Clinical Epidemiology, and Value in Health. Nevertheless, the presence of meta-analyses in survey methodology seems to be slowly increasing. Among the manuscripts identified by this review, the number of publications remains low until 1998 (a total of 12 manuscripts published) and then slowly picks up, with 18 metaanalyses published between 1999 and 2008, and 24 published after 2009 . 
The research gaps in the topics of the existing meta-analyses suggest that out of the seven dimensions of the TSE paradigm, thematic areas under five of them are completely non-covered by research questions of meta-analyses (Online Appendix Tables A5 and A6). For the remaining two TSE dimensions that are covered (measurement and nonresponse error), not all possible intervention and non-experimental moderator variables referring to various study design characteristics are addressed as those affecting measurement or nonresponse error. The moderator variables that were addressed include quality measures for the whole survey, sample characteristics, contacting protocols, the mode of administration, questionnaire design, target population type, and survey setting. For example, studies were found that deal with the effect of the mode of administration on a nonresponse measure.

Correspondingly, there are many unexplored issues representing a large potential for future metaanalytic studies. The most profound research gaps exist in the five TSE dimensions (specification, processing, coverage, sampling, and adjustment error), where no meta-analyses have been identified thus far. Furthermore, for the measurement and nonresponse error TSE dimensions, which are addressed with meta-analyses, almost none of the characteristics of the survey design that are not under the researcher's control have been analyzed (i.e., social environment, technological environment, researcher-respondent interaction, and respondent characteristics; e.g., Groves and Couper 1998; Vehovar et al. 2002). An exception are nonexperimental meta-analyses that include country specifics as a non-experimental moderator variable. The lack of meta-analyses studying the effect on characteristics not under the researcher's control is somewhat understandable, as the researcher cannot really intervene with these dimensions, and thus, they are not included in (experimental) empirical studies. 
Among the characteristics that are under the researcher's control, their effect on measurement and nonresponse issues has not been comprehensively studied. None of the meta-analyses address the effect of the type of survey (panel, cross-sectional, and longitudinal) and interviewer-related issues. In addition, issues that are partially unexplored include contacting protocols in relation to the measurement error dimension, as well as survey characteristics related to the survey setting, sample characteristics, data quality, and format of questions, which are covered by a modest number of meta-analytic studies.

The full scope of the blind spots suggested by this systematic review is theoretical, and there are possible reasons for the limited coverage of some survey methodology topics with meta-analyses suggested by the findings. On the one hand, studying the effect of some survey design characteristics on certain TSE dimensions might not make sense within the framework of survey methodology, as presented by examples in the findings section. On the other hand, for the combinations of TSE dimensions and survey design characteristics where studying the effect is suitable, meta-analyses are limited by the number of available primary studies. Whether there are enough primary studies available could be investigated only by scoping literature searches and performing power analyses for the topics of interest, which is beyond the scope of this paper. Furthermore, many survey methodology studies on the same topic possess differences in terms of replicability, which also limits the possibility of performing a meta-analysis. In addition, the interest of survey methodologists in performing meta-analyses might be limited due to the need for extensive, systematic, and precise desk research of existing literature, which might not be as exciting as planning and implementing new experimental research. 
Further research with the purpose of popularizing meta-analyses in survey methodology, in addition to this systematic review, thus is needed. Such research might go into the direction of showing the importance and centrality of manuscripts reporting on meta-analyses for the further development of knowledge on survey research operations contributing to higher survey data quality. Apart from addressing the two research questions, this systematic review also shows differences in the reporting styles used in meta-analyses with implications for transparency and replicability. The reporting approaches of 16.7 percent of meta-analytic manuscripts convey enough information for a PRISMA or comparable diagram, while the remaining manuscripts lack information to describe all stages of the literature search and selection. Many of these manuscripts were prepared before such standards were established, and the reporting approaches are improving in newer studies. Nevertheless, differences remain, which calls for continued efforts in promoting reporting standards, formal recommendations, and requirements to encourage researchers to provide more details when reporting meta-analytic results.

\section{Supplementary Data}

Supplementary data are freely available online at http://poq.oxfordjournals.org/.

\section{References}

American Psychological Association. 2009. Publication Manual of the American Psychological Association. 6th ed. Washington, DC: American Psychological Association. 
Armstrong, J. Scott, and Edward J. Lusk. 1987. Return Postage in Mail Surveys: A MetaAnalysis. Public Opinion Quarterly 51:233-48. Available at: http://poq.oxfordjournals. org/content/51/2/233.abstract.

Biemer, Paul P. 2010. Total Survey Error: Design, Implementation, and Evaluation. Public Opinion Quarterly 74:817-48. Available at: http://poq.oxfordjournals.org/content/ 74/5/817.abstract.

Biemer, Paul P., and Lars E. Lyberg. 2003. Introduction to Survey Quality. Hoboken, NJ: Wiley. Borenstein, Michael, Larry V. Hedges, Julian P. T. Higgins, and Hannah R. Rothstein. 2009. Introduction to Meta-Analysis. Statistics in Practice. Chichester, UK: Wiley.

Borman, Geoffrey D., and Jeffrey A. Grigg. 2009. Visual and Narrative Interpretation. In The Handbook of Research Synthesis and Meta-Analysis, edited by Harris M. Cooper, Larry V. Hedges, and Jeff C. Valentine, 497-519. New York: Russell Sage Foundation.

Bosnjak, Michael. 2017. Mixed-Mode Surveys and Data Quality. In Methodische Probleme von Mixed-Mode-Ansätzen in der Umfrageforschung, edited by Stefanie Eifler and Frank Faulbaum, 11-25. Springer Fachmedien Wiesbaden. Available at: http://link.springer.com/ chapter/10.1007/978-3-658-15834-7_1.

Brüggen, Elisabeth, Jan van den Brakel, and Jon A. Krosnick. 2016. Establishing the Accuracy of Online Panels for Survey Research. Statistics Netherlands. Available at: https://www.cbs.nl/ en-gb/background/2016/15/establishing-the-accuracy-of-online-panels-for-survey-research.

Cho, Young Ik. 2008. Intercoder Reliability. In Encyclopedia of Survey Research Methods, edited by Paul Lavrakas, 344-45. Thousand Oaks, CA: Sage Publications, Inc. Available at: http://methods.sagepub.com/reference/encyclopedia-of-survey-research-methods/n228.xml. 
Church, Allan H. 1993. Estimating the Effect of Incentives on Mail Survey Response Rates: A Meta-Analysis. Public Opinion Quarterly 57:62-79. Available at: http://poq. oxfordjournals.org/content/57/1/62.short.

de Leeuw, Edith D. 1992. Empirical Evidence of Mode Effects: A Meta-Analysis. In Data Quality in Mail, Telephone and Face to Face Surveys, 21-33. Amsterdam: T. T. Publikaties. Available at: http://eric.ed.gov/?id=ED374136.

de Leeuw, Edith D., and Johannes van der Zouwen. 1988. Data Quality in Telephone and Face to Face Surveys: A Comparative Meta-Analysis. In Telephone Survey Methodology, edited by Robert M. Groves, Paul P. Biemer, Lars E. Lyberg, James T. Massey, William L. Nicholls II, and Joseph Waksberg. New York, NY: Wiley.

Dearholt, Sandra, and Deborah Dang. 2012. Johns Hopkins Nursing Evidence-Based Practice: Models and Guidelines. Indianapolis, IN: Sigma Theta Tau.

Dillman, Don A., Jolene D. Smyth, and Leah M. Christian. 2014. Internet, Phone, Mail, and Mixed-Mode Surveys. The Tailored Design Method. Fourth edition. Hoboken: Wiley.

Glass, Gene V. 1976. Primary, Secondary, and Meta-Analysis of Research. Educational Researcher 5 (10):3-8. Available at: http://www.jstor.org/stable/1174772.

Gnambs, Timo, and Kai Kaspar. 2016. Socially Desirable Responding in Web-Based Questionnaires: A Meta-Analytic Review of the Candor Hypothesis. Assessment. Advance online publication. doi: 10.3758/s13428-014-0533-4.

Goyder, John, Jean Lock, and Trish McNair. 1992. Urbanization Effects on Survey Nonresponse: A Test within and across Cities. Quality and Quantity 26:39-48. Available at: https://link. springer.com/article/10.1007/BF00177996.

Groves, Robert M., and Mick P. Couper. 1998. Nonresponse in Household Interview Surveys. New York, NY: Wiley. 
Groves, Robert M., Floyd J. Fowler Jr, Mick P. Couper, James M. Lepkowski, Eleanor Singer, and Roger Tourangeau. 2009. Survey Methodology. Hoboken, NJ: John Wiley \& Sons.

Groves, Robert M., and Lars E. Lyberg. 2010. Total Survey Error: Past, Present, and Future. Public Opinion Quarterly 74:849-79. Available at: http://poq.oxfordjournals.org/content/ 74/5/849.abstract.

Haidich, Anna-Bettina. 2010. Meta-Analysis in Medical Research. Hippokratia 14 (Suppl 1):29_ 37. Available at: https://www.ncbi.nlm.nih.gov/pmc/articles/PMC3049418/.

Hallyburton, Ann, and Lori A. Evarts. 2014. Gender and Online Health Information Seeking: A Five Survey Meta-Analysis. Journal of Consumer Health on the Internet 18:128-42. Available at: http://www.tandfonline.com/doi/abs/10.1080/15398285.2014.902268.

Hopkins, Kenneth D., and Arlen R: Gullickson. 1992. Response Rates in Survey Research: A Meta-Analysis of the Effects of Monetary Gratuities. Journal of Experimental Education 61:52-62. Available at: http://www.jstor.org/stable/20152353.

Houser, Janet, and Kathleen S. Oman. 2010. Evidence-Based Practice. Sudbury, MA: Jones \& Bartlett Learning.

Jones, Miranda R., Maria Tellez-Plaza, and Ana Navas-Acien. 2013. Smoking, Menthol Cigarettes and All-Cause, Cancer and Cardiovascular Mortality: Evidence from the National Health and Nutrition Examination Survey (NHANES) and a Meta-Analysis. PLOS ONE 8 (10):e77941. Available at: http://journals.plos.org/plosone/article?id=10.1371/journal. pone.0077941.

Koricheva, Julia, and Jessica Gurevitch. 2013. Place of Meta-Analysis among Other Methods of Research Synthesis. In Handbook of Meta-Analysis in Ecology and Evolution, edited by Julia Koricheva, Jessica Gurevitch, and Kerrie Mengersen, 3-13. Princeton, NJ: Princeton University Press. 
Lensvelt-Mulders, Gerty J. L. M., Joop J. Hox, Peter G. M. van der Heuden, and Cora J. M. Maas. 2005. Meta-Analysis of Randomized Response Research Thirty-Five Years of Validation. Sociological Methods \& Research 33:319-48. Available at: http://smr.sagepub. com/content/33/3/319.

Littell, Julia H., Jacqueline Corcoran, and Vijayan Pillai. 2008. Systematic Reviews and MetaAnalysis. Pocket Guides to Social Work Research Methods. New York, NY: Oxford University Press, USA.

Mavletova, Aigul, and Mick P. Couper. 2015. A Meta-Analysis of Breakoff Rates in Mobile Web Surveys. In Mobile Research Methods: Opportunities and Challenges of Mobile Research Methodologies, edited by Daniele Toninelli, Robert Pinter, and Pablo de Pedraza, 81-98. London: Ubiquity Press.

Moher, David, Alessandro Liberati, Jennifer Tetzlaff, Douglas G. Altman, and The PRISMA Group. 2009. Preferred Reporting Items for Systematic Reviews and Meta-Analyses: The PRISMA Statement. PLOS Medicine 6 (7):e1000097. Available at: http://journals.plos.org/ plosmedicine/article?id=10.1371/journal.pmed.1000097.

Mohorko, Anja, Edith Desirée De Leeuw, and Joop J. Hox. 2013. Internet Coverage and Coverage Bias in Europe: Developments across Countries and over Time. Journal of Official Statistics 29:609-22. Available at: https://www.degruyter.com/view/j/jos.2013.29.issue-4/jos2013-0042/jos-2013-0042.xml.

Peeters, Yvette, and Anne M. Stiggelbout. 2010. Health State Valuations of Patients and the General Public Analytically Compared: A Meta-Analytical Comparison of Patient and Population Health State Utilities. Value in Health 13:306-9. Available at: http://www. sciencedirect.com/science/article/pii/S1098301510603762. 
Pigott, T. 2012. Advances in Meta-Analysis. Statistics for Social and Behavioral Sciences. New York, NY: Springer.

Shih, Tse-Hua, and Xitao Fan. 2007. Response Rates and Mode Preferences in Web-Mail MixedMode Surveys : A Meta-Analysis. International Journal of Internet Science 2:59-82. Available at: http://www.ijis.net/ijis2_1/ijis2_1_shih.pdf.

Tourangeau, Roger, and Ting Yan. 2007. Sensitive Questions in Surveys. Psychological Bulletin 133:859-83. Available at: https://www.ncbi.nlm.nih.gov/pubmed/17723033.

Vehovar, Vasja, Zenel Batagelj, Katja Lozar Manfreda, and Metka Zaletel. 2002. Nonresponse in Web Suveys. In Survey Nonresponse, edited by Robert M. Groves, Don A. Dillman, John L. Eltinge, and Roderick J. A. Little, 229-42. New York: Wiley.

Vehovar, Vasja, Katja Lozar Manfreda, and Zenel Batagelj. 1999. Web Surveys: Can The Weighting Solve The Problem? In Proceedings of the Survey Research Methods Section, American Statistical Association, 962-67. Available at: http://www.websm.org/db/12/832/. Yammarino, Francis J., Steven J. Skinner, and Terry L. Childers. 1991. Understanding Mail Survey Response Behavior a Meta-Analysis. Public Opinion Quarterly 55:613-39. Available at: http://poq.oxfordjournals.org/content/55/4/613.

Figure 1. PRISMA Flow Chart of the Manuscript Selection Process in the Systematic Review 
Table 1. Meta-analysis Manuscripts Following Reporting Standards as Recommended by the PRISMA Statement $(N=54)$

\begin{tabular}{lcc}
\hline Search Reporting Level & $\%$ & $\mathrm{n}$ \\
\hline Level 0 (insufficient): reporting only the final number of eligible papers & $42.6 \%$ & 23 \\
$\begin{array}{l}\text { Level 1 (minimal): additionally reporting at least one other metric (e.g., the number of } \\
\text { initial results) but not enough to construct a PRISMA or comparable chart }\end{array}$ & $40.7 \%$ & 22 \\
$\begin{array}{l}\text { Level 2 (comprehensive): sufficient information to construct a PRISMA or comparable } \\
\text { chart }\end{array}$ & $16.7 \%$ & 9 \\
Total & $100.0 \%$ & 54 \\
\hline
\end{tabular}


Table 2. Type of Effect Size Model $(N=91)$

\begin{tabular}{|c|c|c|c|c|c|c|}
\hline \multirow[b]{2}{*}{ Effect Size Model } & \multirow[b]{2}{*}{ Total } & \multicolumn{2}{|c|}{ Meta-analytic Designa } & \multicolumn{3}{|c|}{ Age of Manuscript (years) } \\
\hline & & $\mathrm{E}$ & $\mathrm{N}-\mathrm{E}$ & Mean & Median & St. dev. \\
\hline Random-effects model & $\begin{array}{c}56 \\
61.5 \%\end{array}$ & $\begin{array}{c}32 \\
64.0 \%\end{array}$ & $\begin{array}{c}24 \\
58.5 \%\end{array}$ & 9.4 & 9.0 & 6.3 \\
\hline Fixed-effect model & $\begin{array}{c}35 \\
38.5 \%\end{array}$ & $\begin{array}{c}18 \\
36.0 \%\end{array}$ & $\begin{array}{c}17 \\
41.5 \%\end{array}$ & 21.2 & 26.0 & 9.7 \\
\hline Total & $\begin{array}{c}91 \\
100.0 \%\end{array}$ & $\begin{array}{c}50 \\
100.0 \%\end{array}$ & $\begin{array}{c}41 \\
100.0 \%\end{array}$ & 13.9 & 11.0 & 9.7 \\
\hline
\end{tabular}

a E stands for an experimental meta-analytic design and N-E for a non-experimental meta-analytic design. 
Table 3. Type of Effect Size Measure $(N=91)$

\begin{tabular}{|c|c|c|c|c|c|c|}
\hline \multirow[b]{2}{*}{ Effect Size Measure } & \multirow[b]{2}{*}{ Total } & \multicolumn{2}{|c|}{ Meta-analytic Designa } & \multicolumn{3}{|c|}{ Age of Manuscript (years) } \\
\hline & & E & $\mathrm{N}-\mathrm{E}$ & Mean & Median & St. dev. \\
\hline Odds/risk ratios & $\begin{array}{c}39 \\
42.9 \%\end{array}$ & $\begin{array}{c}25 \\
50.0 \%\end{array}$ & $\begin{array}{c}14 \\
34.1 \%\end{array}$ & 9.7 & 9.0 & 6.1 \\
\hline Mean difference & $\begin{array}{c}29 \\
31.9 \%\end{array}$ & $\begin{array}{c}24 \\
48.0 \%\end{array}$ & $\begin{array}{c}5 \\
12.2 \%\end{array}$ & 18.5 & 19.0 & 10.9 \\
\hline $\begin{array}{l}\text { Standardized regression } \\
\text { coefficient }\end{array}$ & $\begin{array}{c}13 \\
14.3 \%\end{array}$ & $\begin{array}{c}0 \\
0.0 \%\end{array}$ & $\begin{array}{c}13 \\
31.7 \%\end{array}$ & 12.6 & 11.0 & 9.2 \\
\hline Pearson correlation coefficient & $\begin{array}{c}10 \\
11.0 \%\end{array}$ & $\begin{array}{c}1 \\
2.0 \%\end{array}$ & $\begin{array}{c}9 \\
22.0 \%\end{array}$ & 18.9 & 26.0 & 11.0 \\
\hline Total & $\begin{array}{c}91 \\
100.0 \%\end{array}$ & $\begin{array}{c}50 \\
100.0 \%\end{array}$ & $\begin{array}{c}41 \\
100.0 \%\end{array}$ & 13.9 & 11.0 & 9.7 \\
\hline
\end{tabular}

a E stands for an experimental meta-analytic design and N-E for a non-experimental meta-analytic design. 
Table 4. Thematic Areas of Experimental Meta-Analyses: Frequency of Effect Sizes (Structured by TSE) According to Intervention Variables

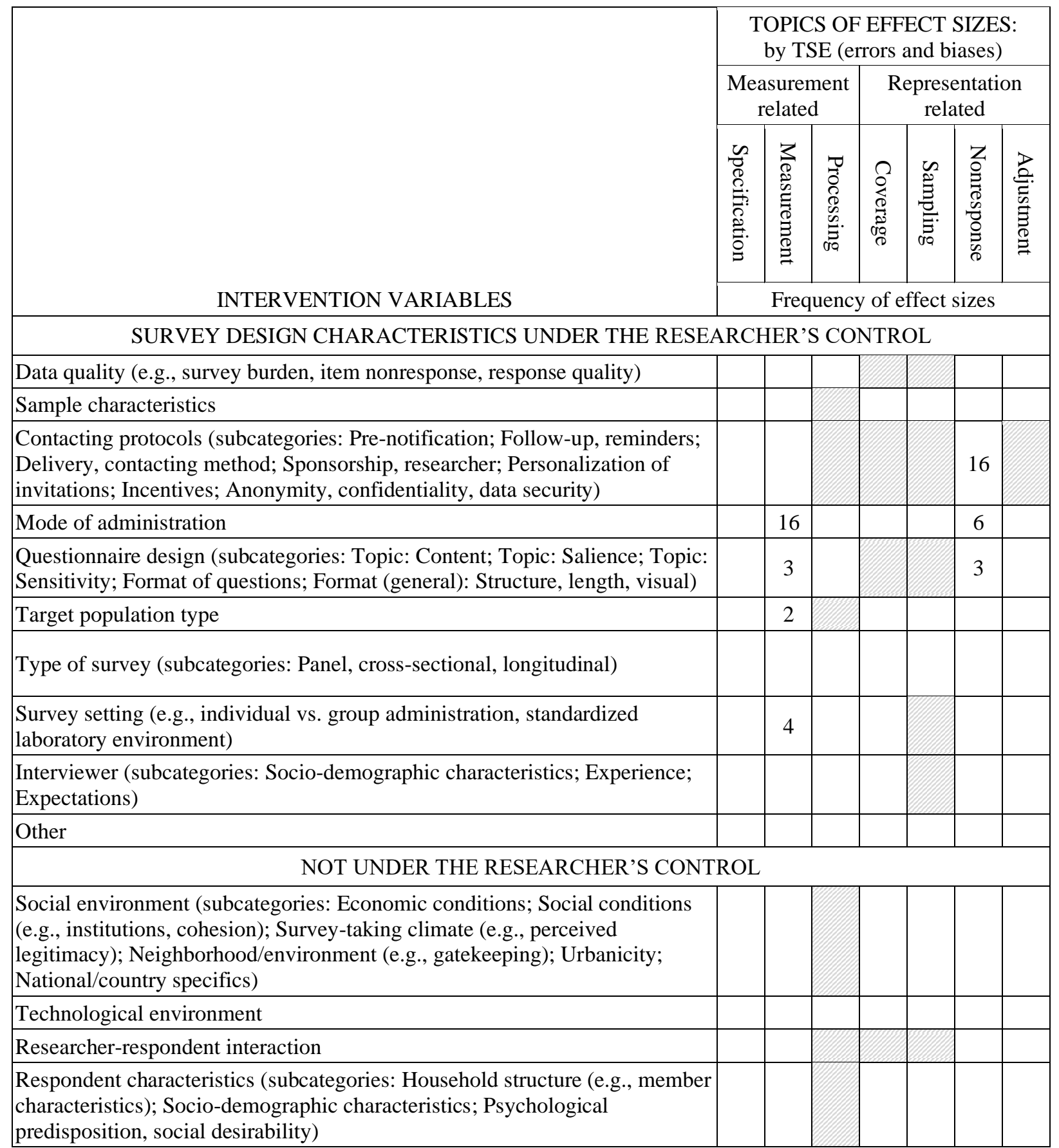

SOURCE.- Values in cells based on analyses by the authors. Category labels for intervention variables are adapted

from Groves and Couper (1998) and Vehovar et al. (2002). Category labels for topic variables are adapted from

Groves et al. (2009) and Biemer (2010). 
Table 5. Thematic Areas of Non-Experimental Meta-Analyses: Frequency of Effect Sizes

\section{(Structured by TSE) According to Non-Experimental Moderator Variables}

\begin{tabular}{|c|c|c|c|c|c|c|c|}
\hline \multirow{4}{*}{ NON-EXPERIMENTAL MODERATOR VARIABLES } & \multicolumn{7}{|c|}{$\begin{array}{l}\text { TOPICS OF EFFECT SIZES: } \\
\text { by TSE (errors and biases) }\end{array}$} \\
\hline & \multicolumn{3}{|c|}{$\begin{array}{l}\text { Measurement } \\
\text { related }\end{array}$} & \multicolumn{4}{|c|}{$\begin{array}{l}\text { Representation } \\
\text { related }\end{array}$} \\
\hline & 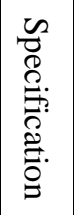 & 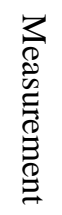 & 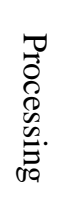 & 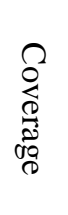 & 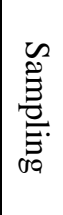 & $\begin{array}{l}Z \\
\stackrel{2}{O} \\
0 \\
0 \\
0 \\
0 \\
0 \\
0 \\
0\end{array}$ & 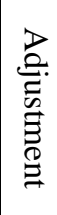 \\
\hline & \multicolumn{7}{|c|}{ Frequency of effect sizes } \\
\hline \multicolumn{8}{|c|}{ SURVEY DESIGN CHARACTERISTICS UNDER THE RESEARCHER'S CONTROL } \\
\hline Data quality (e.g., survey burden, item nonresponse, response quality) & & 3 & & & & 4 & \\
\hline Sample characteristics & & 4 & & & & 3 & \\
\hline $\begin{array}{l}\text { Contacting protocols (subcategories: Pre-notification; Follow-up, reminders; } \\
\text { Delivery, contacting method; Sponsorship, researcher; Personalization of } \\
\text { invitations; Incentives; Anonymity, confidentiality, data security) }\end{array}$ & & 3 & & & & 18 & \\
\hline Mode of administration & & 13 & & & & 9 & \\
\hline $\begin{array}{l}\text { Questionnaire design (subcategories: Topic: Content; Topic: Salience; Topic: } \\
\text { Sensitivity; Format of questions; Format (general): Structure, length, visual) }\end{array}$ & & 12 & & & & 14 & \\
\hline Target population type & & 14 & & & & 6 & \\
\hline \multicolumn{8}{|l|}{ Type of survey (subcategories: Panel, cross-sectional, longitudinal) } \\
\hline $\begin{array}{l}\text { Survey setting (e.g., individual vs. group administration, standardized } \\
\text { laboratory environment) }\end{array}$ & & 7 & & & & 5 & \\
\hline \multicolumn{8}{|l|}{$\begin{array}{l}\text { Interviewer (subcategories: Socio-demographic characteristics; Experience; } \\
\text { Expectations) }\end{array}$} \\
\hline Other & & 4 & & & & 5 & \\
\hline \multicolumn{8}{|l|}{ NOT UNDER THE RESEARCHER'S CONTROL } \\
\hline $\begin{array}{l}\text { Social environment (subcategories: Economic conditions; Social conditions } \\
\text { (e.g., institutions, cohesion); Survey-taking climate (e.g., perceived } \\
\text { legitimacy); Neighborhood/environment (e.g., gatekeeping); Urbanicity; } \\
\text { National/country specifics) }\end{array}$ & & 5 & & & & 1 & \\
\hline \multicolumn{8}{|l|}{ Technological environment } \\
\hline \multicolumn{8}{|l|}{ Researcher-respondent interaction } \\
\hline $\begin{array}{l}\text { Respondent characteristics (subcategories: Household structure (e.g., member } \\
\text { characteristics); Socio-demographic characteristics; Psychological } \\
\text { predisposition, social desirability) }\end{array}$ & & & & & & & \\
\hline
\end{tabular}

SOURCE. - Values in cells based on analyses by the authors. Category labels for intervention variables are adapted

from Groves and Couper (1998) and Vehovar et al. (2002). Category labels for topic variables are adapted from

Groves et al. (2009) and Biemer (2010). 
